\title{
RESOLVED MULTIFREQUENCY RADIO OBSERVATIONS OF GG Tau
}

\author{
Sean M. Andrews ${ }^{1}$, Clatre J. Chandler ${ }^{2}$, Andrea Isella $^{3}$, T. Birnstiel ${ }^{1}$, K. A. Rosenfeld ${ }^{1}$, D. J. Wilner ${ }^{1}$, L. M. Pérez ${ }^{2}$, \\ L. Ricci ${ }^{3}$, J. M. Carpenter ${ }^{3}$, N. Calvet ${ }^{4}$, S. A. Corder ${ }^{5}$, A. T. Deller ${ }^{6}$, C. P. Dullemond ${ }^{7}$, J. S. Greaves ${ }^{8}$, R. J. Harris ${ }^{9}$, \\ Th. Henning ${ }^{10}$, W. Kwon ${ }^{11}$, J. Lazio ${ }^{12}$, H. Linz ${ }^{10}$, L. G. Mundy ${ }^{13}$, A. I. Sargent ${ }^{3}$, S. Storm ${ }^{13}$, And L. Testi ${ }^{14,15}$ \\ ${ }^{1}$ Harvard-Smithsonian Center for Astrophysics, 60 Garden Street, Cambridge, MA 02138, USA; sandrews@ @fa.harvard.edu \\ ${ }^{2}$ National Radio Astronomy Observatory, P.O. Box O, Socorro, NM 87801, USA \\ ${ }^{3}$ California Institute of Technology, 1200 East California Boulevard, Pasadena, CA 91125, USA \\ ${ }^{4}$ Department of Astronomy, University of Michigan, 500 Church Street, Ann Arbor, MI 48109, USA \\ 5 Joint ALMA Observatory, Avenida Alonso de Córdova 3107, Vitacura, Santiago, Chile \\ ${ }^{6}$ The Netherlands Institute for Radio Astronomy (ASTRON), 7990-AA Dwingeloo, The Netherlands \\ ${ }^{7}$ Heidelberg University, Center for Astronomy, Albert Ueberle Str 2, D-69120 Heidelberg, Germany \\ ${ }^{8}$ University of St. Andrews, Physics and Astronomy, North Haugh, St. Andrews KY16 9SS, UK \\ ${ }^{9}$ Department of Astronony, University of Illinois, Urbana, IL 61810, USA \\ ${ }^{10}$ Max Planck Institut für Astronomie, Königstuhl 17, D-69117 Heidelberg, Germany \\ ${ }^{11}$ SRON Netherlands Institute for Space Research, Landleven 12, 9747 AD Groningen, The Netherlands \\ 12 Jet Propulsion Laboratory, California Institute of Technology, 4800 Oak Grove Drive, Pasadena, CA 91106, USA \\ ${ }^{13}$ Department of Astronomy, University of Maryland, College Park, MD 20742, USA \\ ${ }^{14}$ European Southern Observatory, Karl Schwarzschild Str 2, D-85748, Garching, Germany \\ ${ }^{15}$ INAF-Osservatorio Astrofisico di Arcetri, Largo E. Fermi 5, I-50125 Firenze, Italy \\ Received 2014 March 9; accepted 2014 April 22; published 2014 May 13
}

\begin{abstract}
We present subarcsecond resolution observations of continuum emission associated with the GG Tau quadruple star system at wavelengths of 1.3, 2.8, 7.3, and $50 \mathrm{~mm}$. These data confirm that the GG Tau A binary is encircled by a circumbinary ring at a radius of 235 AU with a FWHM width of $\sim 60 \mathrm{AU}$. We find no clear evidence for a radial gradient in the spectral shape of the ring, suggesting that the particle size distribution is spatially homogeneous on angular scales $\gtrsim 0$ '. 1 . A central point source, likely associated with the primary component (GG Tau Aa), exhibits a composite spectrum from dust and free-free emission. Faint emission at $7.3 \mathrm{~mm}$ is observed toward the low-mass star GG Tau Ba, although its origin remains uncertain. Using these measurements of the resolved, multifrequency emission structure of the GG Tau A system, models of the far-infrared to radio spectrum are developed to place constraints on the grain size distribution and dust mass in the circumbinary ring. The non-negligible curvature present in the ring spectrum implies a maximum particle size of 1-10 mm, although we are unable to place strong constraints on the distribution shape. The corresponding dust mass is 30-300 $M_{\oplus}$, at a temperature of 20-30 K. We discuss how this significant concentration of relatively large particles in a narrow ring at a large radius might be produced in a local region of higher gas pressures (i.e., a particle "trap") located near the inner edge of the circumbinary disk.
\end{abstract}

Key words: dust, extinction - protoplanetary disks - radio continuum: planetary systems - stars: individual (GG Tau)

Online-only material: color figures

\section{INTRODUCTION}

The first step of planet formation-the collisional growth of micron-sized dust grains into $>$ kilometer-sized planetesimals, the building blocks of terrestrial planets and the cores of giant planets - is fundamental, but physically complicated and fraught with theoretical uncertainty. A substantial effort with numerical simulations and laboratory experiments is converging on a basic model framework for the growth and migration of solids embedded in a protoplanetary gas disk (see the recent reviews by Testi et al. 2014; Johansen et al. 2014), but direct astronomical observations of these solids are required to test and refine it. Thermal continuum emission at millimeter/radio wavelengths is well-suited for that task, as a (relatively) bright and optically thin tracer of solid particles with sizes up to $\sim 10 \mathrm{~cm}$. The spectral behavior of this emission is diagnostic of the particle size distribution (e.g., Beckwith \& Sargent 1991; Miyake \& Nakagawa 1993; Henning \& Stognienko 1996; D’Alessio et al. 2001; Draine 2006; Ricci et al. 2010b, 2010a). Therefore, spatially resolved measurements of the millimeter/radio "colors" can be used to map out how the particle growth and transport efficiencies vary as a function of the local physical conditions in the gas disk (Isella et al. 2010; Banzatti et al. 2011; Guilloteau et al. 2011; Pérez et al. 2012; Trotta et al. 2013; Menu et al. 2014).

These preliminary studies of the resolved multifrequency continuum emission from disks indicate that the inward radial transport of $\mathrm{mm} / \mathrm{cm}$-sized solids is a crucial factor for explaining the observed color gradients (e.g., Birnstiel et al. 2012). Birnstiel \& Andrews (2014) suggested that this same radial drift, induced by aerodynamic drag on particles that are partially coupled to the gas in its sub-Keplerian velocity field (Adachi et al. 1976; Weidenschilling 1977), is also responsible for the observed discrepancies between the sizes of the line and continuum emission in some disks (e.g., Panić et al. 2009; Andrews et al. 2012; Rosenfeld et al. 2013b). However, there is a fundamental issue with the transport timescales: in these idealized models, radial drift is much too efficient (Takeuchi \& Lin 2002, 2005; Brauer et al. 2007). Perhaps the most promising option for slowing (or stopping) this transport mechanism is with a "bump" in the 
radial gas pressure profile (e.g., Whipple 1972), either locally and stochastically in over-densities generated by turbulence (e.g., Klahr \& Henning 1997; Pinilla et al. 2012b) or globally and with long duration in density concentrations produced near sharp ionization boundaries (just outside a "dead" zone; e.g., Dzyurkevich et al. 2010) or through dynamical interactions with a companion (e.g., Pinilla et al. 2012a).

The most obvious case in which the latter scenario is relevant is for a circumbinary disk, where dynamical interactions between the stars and gas reservoir clear the disk material inside a radius $\sim 3$ times larger than the binary separation (e.g., Artymowicz \& Lubow 1994). The steep gas pressure gradient created by this clearing will trap particles in a circumbinary "ring," which itself might have significantly enhanced pressures due to the dynamical excitation of density waves (e.g., Artymowicz \& Lubow 1996; Kley \& Dirksen 2006; Hayasaki \& Okazaki 2009). GG Tau is the canonical example of a close young stellar pair $(\sim 0$ '.25 projected separation; Leinert et al. 1991; Ghez et al. 1993) with a prominent circumbinary ring, which has been resolved and extensively studied in millimeterwave continuum and molecular line emission (Dutrey et al. 1994; Guilloteau et al. 1999; Piétu et al. 2011) as well as scattered light in the optical and near-infrared (Roddier et al. 1996; Silber et al. 2000; Krist et al. 2002, 2005; McCabe et al. 2002; Itoh et al. 2002; Duchêne et al. 2004). The (unresolved) radio spectrum indicates that $\sim$ millimeter/centimetersized particles are present in the GG Tau circumbinary ring (e.g., Guilloteau et al. 1999; Rodmann et al. 2006; Scaife 2013), lending some additional support to the idea that radial drift is halted near the ring edge.

Here we present resolved measurements of continuum emission from the GG Tau system at wavelengths of 1.3, 2.8, 7.3, and $50 \mathrm{~mm}$, in an effort to characterize the dust population in the GG Tau A circumbinary ring. The observations and data calibration are presented in Section 2. Models of the resolved emission and broadband spectrum are developed and analyzed in Section 3. The results are discussed in the context of current ideas about the evolution of disk solids in Section 4.

\section{OBSERVATIONS AND DATA REDUCTION}

GG Tau was observed with the 15 element $(6 \times 10.4 \mathrm{~m}$ and $9 \times 6.1 \mathrm{~m}$ antennas) Combined Array for Research in Millimeter Astronomy (CARMA) in its $\mathrm{C}$ and $\mathrm{B}$ configurations (30-350 $\mathrm{m}$ and 100-1000 $\mathrm{m}$ baselines, respectively) with the $1 \mathrm{~mm}$ receivers on 2007 September 17 and November 26, and in the B configuration with the $3 \mathrm{~mm}$ receivers on 2008 January 8 , January 17 , and February 15 . In the former, the correlator was set up to process two $500 \mathrm{MHz}$ basebands with coarse spectral resolution in each sideband ( $2 \mathrm{GHz}$ of total bandwidth), with a local oscillator frequency of $228 \mathrm{GHz}(\lambda=1.31 \mathrm{~mm})$. For the latter, a third $500 \mathrm{MHz}$ baseband was added to each sideband ( $3 \mathrm{GHz}$ bandwidth), and the receivers were tuned to $106 \mathrm{GHz}$ $(\lambda=2.83 \mathrm{~mm})$. The observations alternated between GG Tau and the nearby quasars $\mathrm{J} 0530+135$, J0510+180, J0431+206, $\mathrm{J} 0449+113$, and 3C 111, with a source-calibrator cycle time of 12-15 minutes. Additional observations of Uranus were made for calibration purposes. The atmospheric conditions were generally good, with $230 \mathrm{GHz}$ opacities of $\sim 0.15-0.20$ and $0.2-0.3$ during the 1.3 and $2.8 \mathrm{~mm}$ observations, respectively.

The raw visibilities were calibrated and subsequently imaged using standard tasks in the MIRIAD software package. The passband shape across the coarse continuum channels was calibrated using observations of J0530+135, and the (antenna-based) com- plex gain response of the array to both instrumental and atmospheric variations was determined from repeated observations of $\mathrm{J} 0530+135$ or $3 \mathrm{C} 111$. Observations of J0510+180, J0431+206, and J0449+113 were used to assess the quality of the gain calibration; the contribution of decoherence due to small baseline errors and atmospheric phase noise is found to be small, representing a "seeing" disk with FWHM $\leqslant 0$ ". 1 . The absolute amplitude scales were set by bootstrapping J0530+135 flux densities from observations of Uranus, with systematic uncertainties of $\sim 10 \%$. Wideband continuum visibilities were created by averaging the spectra across the sampled passbands. These calibrated continuum visibilities were Fourier inverted assuming natural weighting, deconvolved with the CLEAN algorithm, and restored with a synthesized beam to produce the emission maps shown in Figure 1. The $1.3 \mathrm{~mm}$ visibilities were tapered with a 0 '. 1 FWHM Gaussian kernel before inversion to improve the resulting image quality. The $1.3 \mathrm{~mm}$ continuum map shown in Figure 1 has a $0^{\prime} .67 \times 0$ 0. .57 synthesized beam $\left(\right.$ at P.A. $=132^{\circ}$ ) and an rms noise level of $2.3 \mathrm{mJy}^{\text {beam }}{ }^{-1}$; the corresponding $2.8 \mathrm{~mm}$ map has a $1^{\prime \prime} .19 \times 0.76$ beam $\left(\right.$ at P.A. $\left.=118^{\circ}\right)$ and an rms noise level of $0.5 \mathrm{mJy}^{\text {beam }}{ }^{-1}$.

New observations of the GG Tau field were also made as part of the "Disks@EVLA" key project (project code AC982) with the 27 element ( $25 \mathrm{~m}$ diameter antennas) Karl G. Jansky Very Large Array (VLA), employing the $Q$-band receivers in the C configuration ( $35 \mathrm{~m}$ to $3.4 \mathrm{~km}$ baselines) on 2010 November 27 , and the $C$-band receivers in the A configuration (0.7-36.4 km baselines) on 2011 July 26. For the $Q$-band observations, the recently upgraded correlator was configured to process two contiguous $1 \mathrm{GHz}$ basebands centered around $41.1 \mathrm{GHz}(\lambda=7.29 \mathrm{~mm})$, each comprising eight $128 \mathrm{MHz}$ wide spectral windows with 64 channels. The $C$-band correlator configuration had a similar setup, with the $1 \mathrm{GHz}$ basebands centered at 4.5 and $7.5 \mathrm{GHz}$, for a mean frequency of $6 \mathrm{GHz}$ $(\lambda=5.0 \mathrm{~cm})$. The observations cycled between GG Tau and the nearby calibrator J0431+2037 at $\sim 3$ and 10 minute intervals for the $Q$ - and $C$-bands, respectively. The bright quasars $3 \mathrm{C} 84$ and 3C 147 were also observed for calibration purposes.

The raw visibilities were calibrated and imaged using the "Disks@EVLA" pipeline in the CASA software package (now the standard pipeline for high frequency VLA observations ${ }^{16}$ ). After flagging the data for radio frequency interference and other minor issues, the observations of 3C 84 were used to calibrate the spectral bandpass response of the system after bootstrapping flux densities in each spectral window from observations of 3C 147 (to properly treat the shape of the 3C 84 spectrum over the wide relative bandwidth). The $Q$-band visibilities were then spectrally averaged into 16 pseudo-continuum sub-bands (one per $128 \mathrm{MHz}$ spectral window); the $C$-band data were not averaged, to minimize bandwidth-smearing. Complex gain variations were calibrated with the frequent observations of $\mathrm{J} 0431+2037$, and the absolute amplitude scale was determined using a frequency-dependent emission model for the standard flux density calibrator 3C 147 (Perley \& Butler 2013). The systematic uncertainty in the amplitude scale is $\sim 10 \%$ at $Q$-band and $5 \%$ at $C$-band. The calibrated visibilities were Fourier inverted assuming natural weighting, deconvolved with the multi-frequency synthesis version of CLEAN, and restored with a synthesized beam to make the composite continuum maps shown in Figure 1 . The $Q$-band map has a $0.86 \times 0.61$ synthesized beam $\left(\right.$ at P.A. $=127^{\circ}$ ) and an rms noise level of

\footnotetext{
16 See https://science.nrao.edu/facilities/vla/data-processing/pipeline.
} 


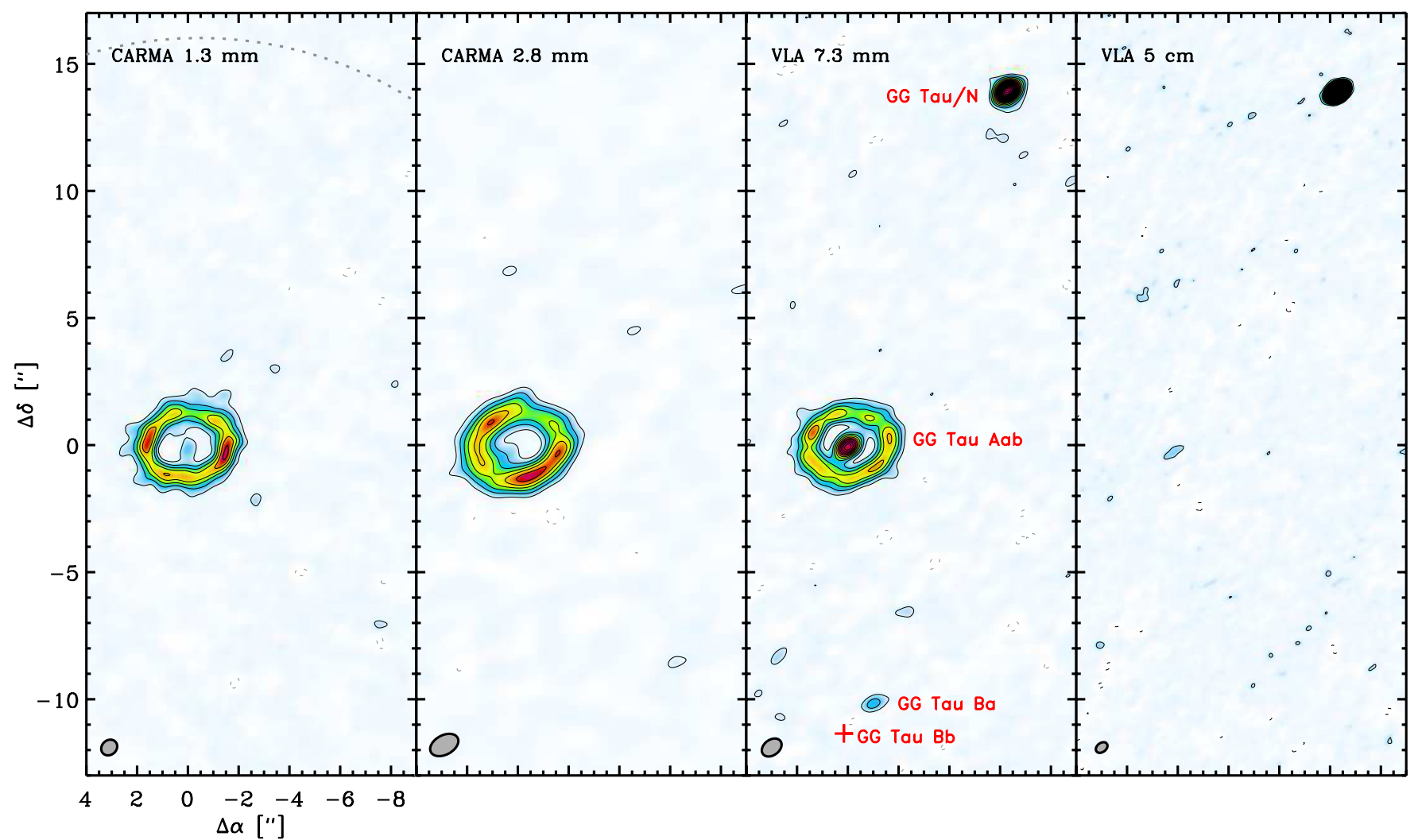

Figure 1. Synthesized continuum images of the GG Tau field at (from left to right) wavelengths of $1.3,2.8,7.3$, and $50 \mathrm{~mm}$. Contours are drawn at $3 \sigma$ intervals in each panel $\left(7,1.5,0.04\right.$, and $0.02 \mathrm{mJy}_{\text {beam }}{ }^{-1}$ from left to right), and synthesized beam dimensions are marked in the lower left corners. The primary beam for the CARMA $1.3 \mathrm{~mm}$ image is shown as a dotted gray curve. The key emission components are labeled in the VLA $Q$-band (7.3 mm) image, including the circumbinary dust ring around GG Tau Aab, faint radio emission toward the low-mass binary GG Tau Bab (from component Ba), and the bright radio emission from the background galaxy GG Tau/N.

(A color version of this figure is available in the online journal.)

$13 \mu \mathrm{Jy}$ beam $^{-1}$, and the $C$-band map has a $0{ }^{\prime} 51 \times 00^{\prime \prime} 36$ beam $\left(\right.$ at P.A. $\left.=127^{\circ}\right)$ with an rms noise level of $6 \mu \mathrm{Jy}^{\text {beam }}{ }^{-1}$. The $C$-band image reconstruction required substantial extra care, including imaging of the entire primary beam and faceting on exceptionally bright background sources, to minimize artifacts at the field center. The images shown in Figure 1 are not corrected for the response of the primary beam.

\section{ANALYSIS AND RESULTS}

The multifrequency continuum images in Figure 1 show emission from three distinct components: (1) an extended ring around the close binary GG Tau A at 1.3, 2.8, and $7.3 \mathrm{~mm}$, along with point-like emission at its center detected at 1.3, 7.3, and $50 \mathrm{~mm} ;{ }^{17}$ (2) faint, unresolved emission at $7.3 \mathrm{~mm}$ associated with the low-mass star GG Tau Ba (no emission is found toward its $\sim 1^{\prime \prime} .5$ companion $\mathrm{Bb}$ ); and (3) bright, unresolved emission at 7.3 and $50 \mathrm{~mm}$ from the (presumed) extragalactic interloper GG Tau/N. The composite flux densities, $S_{v}$, or upper limits for each of these components are compiled in Table 1 and displayed together in Figure 2 (note that the measurements for the GG Tau $\mathrm{B}$ and $\mathrm{N}$ components in this figure and table were determined from images that were corrected for the response of the primary beam). For completeness, we also include literature measurements of the radio spectra for each component in this figure.

\footnotetext{
17 It is worth pointing out that we do not detect the "streamer" identified by Piétu et al. (2011); its estimated surface brightness is comparable to the rms noise level in our $1.3 \mathrm{~mm}$ map, and would likely lie well below the noise floor
} at longer wavelengths if its origin is thermal dust emission.
The radio spectral index of GG Tau/N, $\alpha \approx-0.7$ (defined as $S_{v} \propto v^{\alpha}$ ), and its non-detection at millimeter wavelengths indicate a non-thermal emission mechanism (e.g., Bieging et al. 1984). Given this index, GG Tau/N is likely a background extragalactic source (AGN) emitting an optically thin synchrotron spectrum; however, it is worth noting that there is no confirmed counterpart at any optical or infrared wavelength. GG Tau B is a low-mass T Tauri binary (White et al. 1999) with a modest infrared excess (Luhman et al. 2010); the $7.3 \mathrm{~mm}$ emission from the primary (Ba; spectral type M5) found here is the first detection longward of $24 \mu \mathrm{m}$. The nature of this $Q$-band emission from $\mathrm{Ba}$ is not clear, given the non-detections at other wavelengths. The derived $C$-band upper limit allows $\alpha \gtrsim 0.6$, consistent with origins in a magnetically active corona (White et al. 1994; Cranmer et al. 2013) or dense wind (Reynolds 1986). Likewise, non-detections at 1.3 and $2.8 \mathrm{~mm}$ suggest that $\alpha \lesssim 2.3$, which is also commensurate with thermal emission from a relatively cold disk (as might be expected around such a low-mass host star; e.g., Andrews et al. 2013) or optically thin emission from large dust particles (e.g., Ricci et al. 2010b). Variable non-thermal radio emission is an additional (and not mutually exclusive) possibility.

We focus here on the multifrequency, resolved emission structure from GG Tau A, itself composed of a narrow circumbinary ring and a point-like contribution associated with one or both components of the close binary (Dutrey et al. 1994; Guilloteau et al. 1999; Piétu et al. 2011). In the following sections, we describe a simple model to quantify these emission components as a function of the observing frequency, and use those results to 
Table 1

Radio Spectra of GG Tau Components

\begin{tabular}{|c|c|c|c|}
\hline Component & $\begin{array}{c}\lambda \\
(\mathrm{mm})\end{array}$ & $\begin{array}{c}S_{v} \\
(\mathrm{mJy})\end{array}$ & Ref. \\
\hline GG Tau A & $\begin{array}{r}0.10 \\
0.10 \\
0.14 \\
0.15 \\
0.16 \\
0.16 \\
0.18 \\
0.19 \\
0.35 \\
0.44 \\
0.44 \\
0.44 \\
0.62 \\
0.77 \\
0.79 \\
0.79 \\
0.79 \\
0.79 \\
0.87 \\
1.06 \\
1.09 \\
1.09 \\
1.09 \\
1.09 \\
1.12 \\
1.25 \\
1.26 \\
1.26 \\
1.26 \\
1.31 \\
1.33 \\
1.40 \\
1.92 \\
2.68 \\
2.78 \\
2.83 \\
3.06 \\
3.40 \\
6.92 \\
7.29 \\
19.09 \\
50.00\end{array}$ & $\begin{array}{c}5158 \pm 1410 \\
6560 \pm 1469 \\
8995 \pm 2291 \\
7620 \pm 3048 \\
8600 \pm 3440 \\
6076 \pm 1974 \\
8220 \pm 3288 \\
7130 \pm 2853 \\
6528 \pm 1639 \\
4540 \pm 766 \\
4160 \pm 665 \\
2726 \pm 726 \\
1370 \pm 382 \\
1250 \pm 323 \\
1110 \pm 163 \\
1710 \pm 176 \\
1590 \pm 170 \\
1650 \pm 183 \\
1255 \pm 138 \\
800 \pm 206 \\
740 \pm 141 \\
1070 \pm 111 \\
830 \pm 88 \\
850 \pm 90 \\
770 \pm 78 \\
593 \pm 130 \\
690 \pm 75 \\
630 \pm 66 \\
630 \pm 70 \\
558 \pm 58 \\
557 \pm 56 \\
604 \pm 61 \\
320 \pm 68 \\
85 \pm 10 \\
73 \pm 15 \\
79 \pm 9 \\
41 \pm 9 \\
38 \pm 4 \\
3.24 \pm 0.77 \\
2.67 \pm 0.29 \\
0.25 \pm 0.05 \\
0.036 \pm 0.007\end{array}$ & $\begin{array}{l}\text { IRAS } \\
\text { Howard et al. (2013) } \\
\text { AKARI } \\
\text { Howard et al. (2013) } \\
\text { Howard et al. (2013) } \\
\text { AKARI } \\
\text { Howard et al. (2013) } \\
\text { Howard et al. (2013) } \\
\text { Andrews \& Williams (2005) } \\
\text { Moriarty-Schieven \& Butner (1997) } \\
\text { Moriarty-Schieven \& Butner (1997) } \\
\text { Andrews \& Williams (2005) } \\
\text { Beckwith \& Sargent (1991) } \\
\text { Beckwith \& Sargent (1991) } \\
\text { Moriarty-Schieven et al. (1994) } \\
\text { Moriarty-Schieven \& Butner (1997) } \\
\text { Moriarty-Schieven \& Butner (1997) } \\
\text { Moriarty-Schieven \& Butner (1997) } \\
\text { Andrews \& Williams (2005) } \\
\text { Beckwith \& Sargent (1991) } \\
\text { Moriarty-Schieven et al. (1994) } \\
\text { Moriarty-Schieven \& Butner (1997) } \\
\text { Moriarty-Schieven \& Butner (1997) } \\
\text { Moriarty-Schieven \& Butner (1997) } \\
\text { Piétu et al. (2011) } \\
\text { Beckwith et al. (1990) } \\
\text { Moriarty-Schieven \& Butner (1997) } \\
\text { Moriarty-Schieven \& Butner (1997) } \\
\text { Moriarty-Schieven \& Butner (1997) } \\
\text { This paper } \\
\text { Harris et al. (2012) } \\
\text { Guilloteau et al. (1999) } \\
\text { Moriarty-Schieven \& Butner (1997) } \\
\text { Dutrey et al. (1994) } \\
\text { Looney et al. (2000) } \\
\text { This paper } \\
\text { Ohashi et al. (1991) } \\
\text { Guilloteau et al. (1999) } \\
\text { Rodmann et al. (2006) } \\
\text { This paper } \\
\text { Scaife (2013) } \\
\text { This paper }\end{array}$ \\
\hline GG Tau Ba & $\begin{array}{r}1.31 \\
1.33 \\
2.83 \\
7.29 \\
50.00 \\
\end{array}$ & $\begin{array}{c}<9 \\
<7 \\
<1.7 \\
0.058 \pm 0.014 \\
<0.02\end{array}$ & $\begin{array}{l}\text { This paper } \\
\text { Harris et al. (2012) } \\
\text { This paper } \\
\text { This paper } \\
\text { This paper }\end{array}$ \\
\hline GG Tau Bb & $\begin{array}{r}1.31 \\
1.33 \\
2.83 \\
7.29 \\
50.00 \\
\end{array}$ & $\begin{array}{c}<9 \\
<7 \\
<1.7 \\
<0.04 \\
<0.02 \\
\end{array}$ & $\begin{array}{l}\text { This paper } \\
\text { Harris et al. (2012) } \\
\text { This paper } \\
\text { This paper } \\
\text { This paper }\end{array}$ \\
\hline GG Tau/N & $\begin{array}{r}1.31 \\
2.83 \\
7.29 \\
19.09 \\
20.03 \\
50.00 \\
61.37\end{array}$ & $\begin{aligned} &<14 \\
&<2.0 \\
& 0.71 \pm 0.07 \\
& 2.23 \pm 0.12 \\
& 3 \pm 1 \\
& 2.84 \pm 0.14 \\
& 3.7 \pm 0.4\end{aligned}$ & $\begin{array}{l}\text { This paper } \\
\text { This paper } \\
\text { This paper } \\
\text { Scaife (2013) } \\
\text { Bieging et al. (1984) } \\
\text { This paper } \\
\text { Bieging et al. (1984) }\end{array}$ \\
\hline
\end{tabular}

Notes. The uncertainties on the flux densities include both statistical and systematic calibration terms (added in quadrature). Upper limits are quoted at the $3 \sigma$ level, assuming point source emission. The GG Tau B and $\mathrm{N}$ flux densities were determined after correction for the primary beam responses in each observation. 


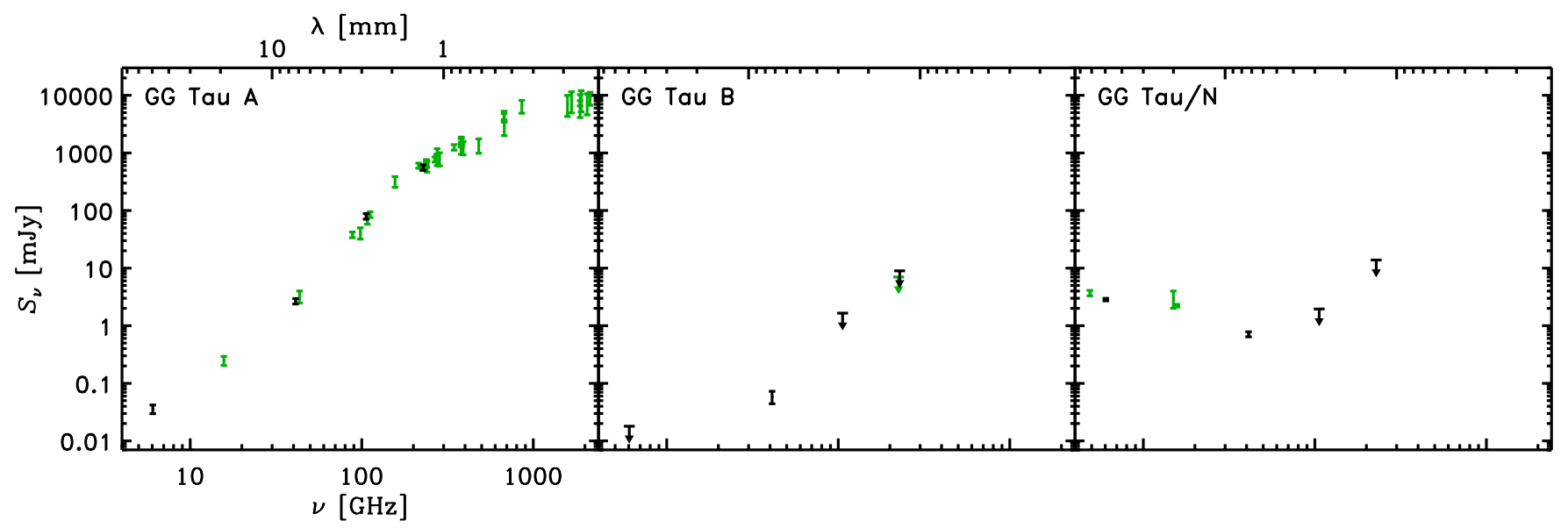

Figure 2. Millimeter/radio spectra for the three emission components in the GG Tau field: A (left; see Section 3.1 for more details), B (middle), and N (right), where the emission from the latter two components were estimated from images that have been corrected for the response of the primary beam. Our measurements are shown in black, and flux densities from the literature are marked in green (Table 1 lists $S_{v}$ for each component). The error bars represent the formal statistical uncertainties and the systematic calibration uncertainties, added in quadrature. Upper limits (at $3 \sigma$ ) are marked with a horizontal line and a downward-pointing arrow.

(A color version of this figure is available in the online journal.)

assess the emission origins. First, we establish the basic spatial structure of the GG Tau A emission (in Section 3.1), and then we employ that resolved information to model the spectrum and extract physical constraints on the properties of the constituent solid particles (e.g., mass, temperature, size distribution; in Section 3.2).

\subsection{Models for Resolved Emission from GG Tau A}

We adopt a simple model prescription for the brightness distribution of the GG Tau A emission consisting of an azimuthally symmetric Gaussian ring and a central point source. The ring is described by seven parameters: a mean radius $\mu_{r}$, width $\sigma_{r}$, integrated flux density $S_{\nu, r}\left(=\int I_{\nu, r} d \Omega\right)$, inclination angle $i\left(0^{\circ}\right.$ is face-on), minor axis position angle $\varphi$ (representing the skyprojected orientation of the ring rotation axis), and two nuisance parameters to account for the ring center location $\left\{\Delta \alpha_{r}, \Delta \delta_{r}\right\}$ (defined as arcsecond offsets in right ascension and declination from the observed phase center). The point source component includes three additional parameters: a flux density $S_{v, c}$ and projected offsets $\left\{\Delta \alpha_{c}, \Delta \delta_{c}\right\}$ relative to the ring center. We assume a distance of $140 \mathrm{pc}$ (e.g., Torres et al. 2009), and compute models for different frequencies independently.

For a given set of these 10 parameters, we calculate synthetic complex visibilities, $V_{v}$, sampled at the same spatial frequencies, $(u, v)$, as the relevant observations. We then evaluate a Cauchy log-likelihood function (cf. Sivia \& Skilling 2006) to represent the probability of the model, $\left\{V_{v}^{\mathrm{M}}(u, v)\right\}$, given the observed complex visibilities, $\left\{V_{v}^{\mathrm{D}}(u, v)\right\}$, and their uncertainties, $\left\{\sigma_{v}^{\mathrm{D}}(u, v)\right\}$,

$$
\begin{aligned}
\mathcal{L} & \propto \sum_{k} \ln \left(\frac{1-e^{-R_{k}^{2} / 2}}{R_{k}^{2}}\right) ; \text { where } \\
R_{k} & =\left(\frac{V_{\nu}^{\mathrm{D}}\left(u_{k}, v_{k}\right)-V_{v}^{\mathrm{M}}\left(u_{k}, v_{k}\right)}{\sigma_{v}^{\mathrm{D}}\left(u_{k}, v_{k}\right)}\right) .
\end{aligned}
$$

This log-likelihood function was preferred over its more familiar Gaussian counterpart (where $\mathcal{L} \propto-\chi^{2} / 2=\sum_{k} R_{k}^{2} / 2$ ) because it is more forgiving of outliers (mitigating parameter bias due to phase calibration systematics on long baselines) and more conservative (which is important, since our error estimates are derived solely from the visibility weights, and therefore do not treat scatter due to issues like pointing errors, atmospheric phase noise, etc.). The posterior probability distribution function (PDF) was sampled with Monte Carlo Markov Chain (MCMC) calculations, using the Goodman \& Weare (2010) ensemble sampler as implemented by Foreman-Mackey et al. (2013).

A set of initial MCMC calculations was conducted with uniform priors on all parameters. However, given the very weak emission from the central point source component at 1.3 and $2.8 \mathrm{~mm}$, convergence on the relative offset parameters $\left\{\Delta \alpha_{c}, \Delta \delta_{c}\right\}$ was prohibitively slow (and could therefore lead us to biased inferences of $S_{v, c}$ ). In these initial calculations, we found that the $1.3 \mathrm{~mm}$ relative offsets were entirely consistent with the well-constrained values at $7.3 \mathrm{~mm}$. Therefore, new MCMC calculations were made with (independent) Gaussian priors on these offsets, centered on the $7.3 \mathrm{~mm}$ values and with standard deviations corresponding to the inferred 68\% marginal widths of their posterior PDFs. In any case, this had no impact on the inferences for other parameters.

Figure 3 is a representation of the sampled posterior PDFs in the form of a staircase diagram, showing both pair-wise parameter covariances (contours are drawn at the 68 and 95\% confidence intervals) and marginalized posterior PDFs for individual parameters (the four offset parameters are not shown, and the flux density parameters are normalized to the peaks of their marginal posterior PDFs, for the sake of clarity). The panel in the upper right corner is a visualization of the (areanormalized) radial surface brightness profiles reconstructed from random draws to the joint posterior PDFs, where the shaded widths of each profile are representative of the $68 \%$ (i.e., $\sim 1 \sigma$ ) confidence intervals. Table 2 summarizes the modeling results, listing the "best-fit" (peaks of the marginal posterior PDFs) parameter values and their $68 \%$ confidence intervals at each observing frequency. A direct comparison of the data and bestfit models is shown in Figure 4, in both the image plane and with the azimuthally averaged (and deprojected) visibility profiles.

Within the uncertainties, the GG Tau A circumbinary ring has the same mean radius and width (as well as inclination, orientation, and center) at all observed frequencies; $\mu_{r} \approx$ 230-240 AU $\left(\sim 1^{\prime \prime} \cdot 7\right)$ and $\sigma_{r} \approx 20-30 \mathrm{AU}$ (corresponding to a FWHM of 0 !'3-0!'5; i.e., it is only marginally resolved). There is a hint that the ring is slightly narrower (at the $\sim 1 \sigma$ 


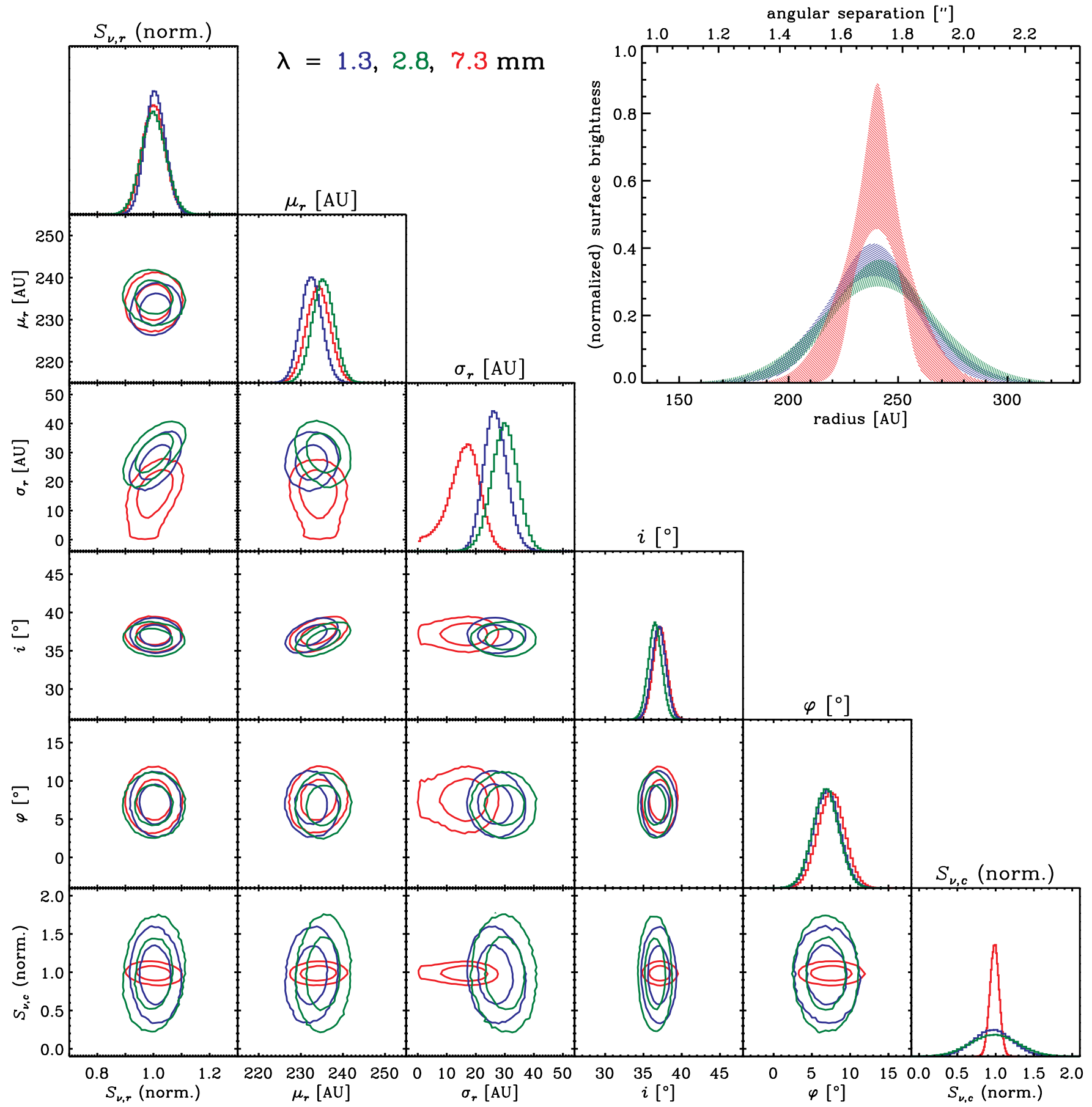

Figure 3. Summary of the posterior PDFs inferred from MCMC calculations in reference to resolved interferometer data at 1.3, 2.8, and 7.3 mm, assuming a model composed of a central point source and a Gaussian ring. The staircase plot to the left shows marginalized two-parameter posterior PDF surfaces, with contours drawn at $68 \%$ and $95 \%$ confidence intervals. Marginalized posterior PDFs for each parameter are shown along the diagonal. The ring and point source flux densities, $S_{v, r}$ and $S_{v, c}$ respectively, are normalized by their best-fit values for clarity. Note that the 4 directional offset parameters $\left(\Delta \alpha_{r}, \Delta \delta_{r}, \Delta \alpha_{c}\right.$, and $\left.\Delta \delta_{c}\right)$ are not shown, to simplify the plot. The top right panel is a visual representation of the (normalized) radial surface brightness profiles derived from this analysis; the widths of these profiles represent the $68 \%$ confidence intervals.

(A color version of this figure is available in the online journal.)

level) at $7.3 \mathrm{~mm}$, although the difference is not statistically (or practically) significant. Taken together, this indicates that the spectral behavior of the dust continuum emission does not vary radially across the ring on the angular scales and at the spectral sensitivity currently available. If we assume that a Gaussian distribution is an appropriate spatial model and a power-law with frequency is a reasonable spectral model, we can very roughly estimate from the fitting results derived here that $\Delta \alpha \lesssim 0.3$ on (radial) angular scales $\gtrsim 0.1$. Variations at finer scales are possible, and perhaps likely (see Section 4).

There is some non-negligible curvature in the ring spectrum, with a steeper spectral index at lower frequencies: $\alpha(41-106 \mathrm{GHz}) \approx 3.7 \pm 0.2$ and $\alpha(106-228 \mathrm{GHz}) \approx 2.6 \pm 0.2$ (the quoted uncertainties account for the $\sim 10 \%$ systematics introduced in the amplitude calibrations). The peak brightness temperature of the ring at $1.3 \mathrm{~mm}$ is only $\sim 0.2 \mathrm{~K}$, confirming that 

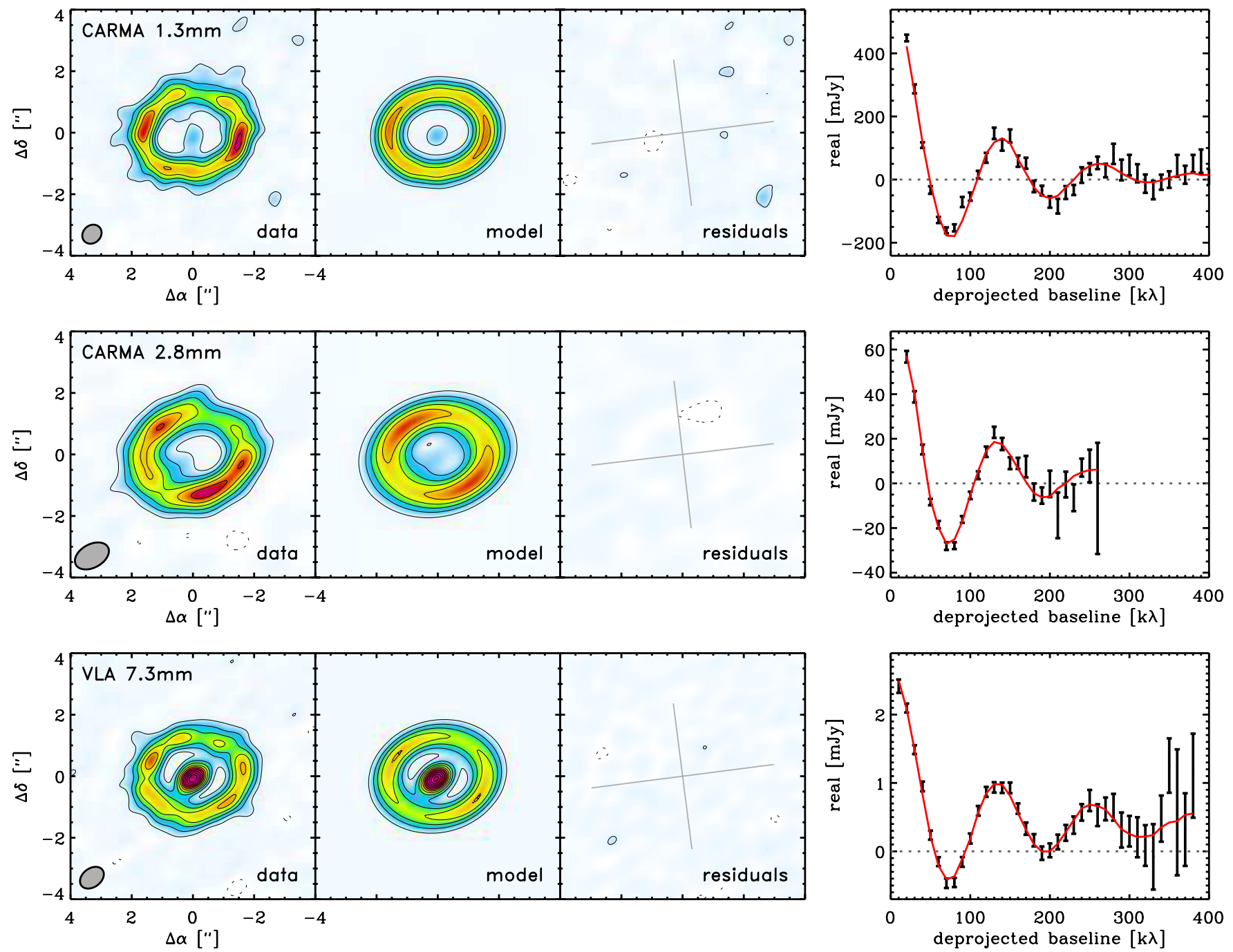

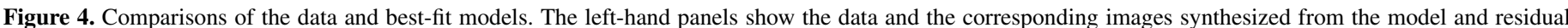

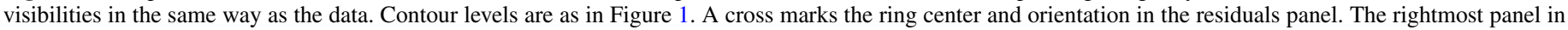
each row shows the azimuthally averaged (real) visibility profiles, deprojected according to the derived ring geometry (data in black, models in red).

(A color version of this figure is available in the online journal.)

Table 2

Inferred Visibility Model Parameters

\begin{tabular}{lccc}
\hline \hline Parameter & $1.3 \mathrm{~mm}$ & $2.8 \mathrm{~mm}$ & $7.3 \mathrm{~mm}$ \\
\hline$S_{v, r}(\mathrm{mJy})$ & $543 \pm 21$ & $77 \pm 4$ & $2.23_{-0.12}^{+0.08}$ \\
$\mu_{r}(\mathrm{AU})$ & $232 \pm 3$ & $235 \pm 3$ & $234 \pm 3$ \\
$\sigma_{r}(\mathrm{AU})$ & $26 \pm 5$ & $29_{-5}^{+4}$ & $17_{-8}^{+4}$ \\
$i\left(^{\circ}\right)$ & $37 \pm 1$ & $37 \pm 1$ & $37 \pm 1$ \\
$\varphi\left(^{\circ}\right)$ & $7 \pm 2$ & $7 \pm 2$ & $7 \pm 2$ \\
$S_{v, c}(\mathrm{mJy})$ & $15_{-7}^{+3}$ & $2.2_{-0.9}^{+0.6}$ & $0.44_{-0.04}^{+0.02}$ \\
$\Delta \alpha_{c}\left(^{\prime \prime}\right)$ & $+0.03 \pm 0.04$ & $+0.06 \pm 0.05$ & $+0.05 \pm 0.04$ \\
$\Delta \delta_{c}\left(^{\prime \prime}\right)$ & $-0.10 \pm 0.05$ & $-0.09 \pm 0.06$ & $-0.08 \pm 0.03$ \\
\hline
\end{tabular}

Notes. The quoted uncertainties correspond to $68 \%(\sim 1 \sigma)$ confidence intervals; the associated $\sim 10 \%$ calibration uncertainty is not applied to the flux density parameters. The nuisance parameters describing the offset of the ring center from the observed phase center are not included.

the emission is optically thin. Therefore, the observed spectral curvature is a by-product of the intrinsic shape of the dust opacity spectrum (and perhaps cool temperatures; see Section 3.2 for more details). The ring is not detected at a wavelength of $5 \mathrm{~cm}$.
Assuming it has the same emission morphology as at shorter wavelengths, a limit on its integrated flux density can be made based on the measured rms noise level $\left(6 \mu \mathrm{Jy} \mathrm{beam}^{-1}\right)$ in the $C$-band map: we estimate $S_{v, r}<40 \mu \mathrm{Jy}(3 \sigma)$.

The central point-like component, originally identified at 1.4 and $1.1 \mathrm{~mm}$ by Guilloteau et al. (1999) and Piétu et al. (2011), is also detected here at 1.3, 7.3, and $50 \mathrm{~mm}$. Models with a relatively faint central source at $2.8 \mathrm{~mm}$ (as listed in Table 2) provide better overall matches to the visibility data, although formally the inference on $S_{v, c}$ is only marginally significant (greater than zero at the $\sim 2.7 \sigma$ level): alternatively, we could quote a $3 \sigma$ upper limit as $S_{v, c}<4.0 \mathrm{mJy}$. The (sparse) radio spectrum of this central source includes contributions from different emission mechanisms. The spectral index at high frequencies is steep, $\alpha(41-228 \mathrm{GHz}) \approx 2.1 \pm 0.2$, and consistent with optically thick thermal emission from a warm dust disk. The low-frequency radio spectrum is considerably more shallow, $\alpha(6-41 \mathrm{GHz}) \approx 1.3 \pm 0.1$; when combined with the thermal spectrum, the radio emission is best explained with an intrinsically flat spectrum $(\alpha \approx 0)$, suggesting a contribution from optically thin free-free radiation. We find no evidence that this central component is resolved: models of the $7.3 \mathrm{~mm}$ 
emission that assume a Gaussian emission distribution (rather than a point source) indicate a radial width $<12 \mathrm{AU}(3 \sigma$; this corresponds to a HWHM $<0$ '.2). However, our models suggest that this component is marginally offset from the ring center, with a sky-projected separation of $90 \pm 30$ mas to the southeast (at P.A. $\approx 149^{\circ} \pm 1^{\circ}$ ) measured from the $7.3 \mathrm{~mm}$ data. Assuming the recent orbit calculations of Köhler (2011), and associating the ring center with the projected binary center of mass (with the $\sim 0.9: 1$ mass ratio of White et al. 1999), these constraints on the scale and orientation of the offsets suggest that the emission likely originates from the primary component GG Tau Aa. A similar inference was made for the $1.1 \mathrm{~mm}$ emission detected by Piétu et al. (2011), based on the orbit calculations of Beust \& Dutrey (2005).

\subsection{Models of the GG Tau A Spectrum}

Having established an empirical model of the resolved multifrequency emission from GG Tau A, we shift focus to develop a more physically motivated model of the entire far-infrared to radio spectrum. The basic goal is to help characterize the dust population in the circumbinary ring. The emission structure of GG Tau A that we derived in the previous section is unusually simple in the context of protoplanetary disks. The circumbinary ring has a well-defined mean radius and width, and is spatially isolated from the central component. The absence of a spatial gradient in the ring spectrum, along with its apparently low optical depth, suggest that the continuum emission is a reasonably good tracer of the dust column density distribution. Moreover, the ring itself is sufficiently narrow and distant from the central heating sources that we expect it should have a near-constant radial temperature profile (irradiation heating would produce a variation of $\lesssim 15 \%$, roughly $2-3 \mathrm{~K}$, across the ring; see below). So unlike a typical disk, where large and uncertain gradients in temperature and density can act as severe obstacles, we have an interesting opportunity to use the $\mathrm{mm} /$ radio spectrum and our structural constraints from the resolved data to extract some constraints on the size distribution of the solids in the circumbinary ring.

To that end, we define a spectrum model as the sum of two components: thermal dust emission in the ring (with flux densities $S_{v, r}$ ) and a composite emission origin associated with the central point source $\left(S_{v, c}\right)$. For the latter, we assume a double power-law spectrum,

$$
S_{v, c}^{\mathrm{M}}=S_{v, 0}^{\text {dust }}\left(\frac{v}{10 \mathrm{GHz}}\right)^{\alpha_{\mathrm{dust}}}+S_{v, 0}^{\mathrm{ff}}\left(\frac{v}{10 \mathrm{GHz}}\right)^{\alpha_{\mathrm{ff}}} .
$$

For the dust emission in the ring, we use the classic and simple one-dimensional thermal continuum model often adopted for disks (Adams et al. 1987; Beckwith et al. 1990),

$$
S_{v, r}^{\mathrm{M}}=\frac{2 \pi \cos i}{d^{2}} \int d r r B_{v}\left(T_{r}\right)\left(1-e^{-\kappa_{\nu} \Sigma_{r} / \cos i}\right),
$$

where $d=140 \mathrm{pc}, i$ is the ring inclination, $B_{v}\left(T_{r}\right)$ is the Planck function at a given temperature, $\Sigma_{r}$ is a Gaussian surface density profile with mean $\mu_{r}$, width $\sigma_{r}$, and peak value $\Sigma_{0}$, and $\kappa_{v}$ is the dust opacity spectrum. Model opacity spectra were derived assuming a power-law grain size $(a)$ distribution, with index $q$ (where $d n / d a \propto a^{-q}$ ) and maximum size $a_{\max }$ (the minimum size was set to $0.1 \mu \mathrm{m}$ ). For easier comparisons with related work, we employed the Ricci et al. (2010b) dust mixture, ${ }^{18}$

\footnotetext{
18 From a material composition standpoint, this mixture is similar to the one advocated by Pollack et al. (1994).
}

with volume fractions of $30 \%$ vacuum (porosity), $7 \%$ silicates, $21 \%$ carbonaceous materials, and $42 \%$ water ice, using optical constants from Weingartner \& Draine (2001), Zubko et al. (1996), and Warren (1984), respectively. The optical constants for the mixture were determined with the Bruggeman rule, and the corresponding $\kappa_{v}$ for any particle size were computed with a Mie code. Altogether, the model has 11 parameters, $\left\{S_{\nu, 0}^{\text {dust }}\right.$, $\left.\alpha_{\text {dust }}, S_{\nu, 0}^{\mathrm{ff}}, \alpha_{\mathrm{ff}}, i, T_{r}, \Sigma_{0}, \mu_{r}, \sigma_{r}, a_{\mathrm{max}}, q\right\}$.

For any parameter combination, we define two residual terms at each frequency that record the fit quality relative to the resolved measurements of the ring and point source flux densities,

$$
R_{v, r}=\left(\frac{S_{v, r}^{\mathrm{D}}-S_{v, r}^{\mathrm{M}}}{\sigma_{v, r}^{\mathrm{D}}}\right) \text { and } R_{\nu, c}=\left(\frac{S_{v, c}^{\mathrm{D}}-S_{\nu, c}^{\mathrm{M}}}{\sigma_{v, c}^{\mathrm{D}}}\right),
$$

respectively; the "observed" flux densities, $S_{v}^{\mathrm{D}}$, and their associated uncertainties, $\sigma_{v}^{\mathrm{D}}$, can be found in Table 2. ${ }^{19}$ Moreover, we made use of the unresolved photometry in the literature to compare with the sum of the model components, with an additional residual term

$$
R_{v, \text { tot }}=\left(\frac{S_{v, \text { tot }}^{\mathrm{D}}-\left[S_{v, r}^{\mathrm{M}}+S_{v, c}^{\mathrm{M}}\right]}{\sigma_{v, \text { tot }}^{\mathrm{D}}}\right)
$$

at each frequency, where $\left\{S_{v, \text { tot }}^{\mathrm{D}}, \sigma_{\nu, \text { tot }}^{\mathrm{D}}\right\}$ are compiled in Table 1. These terms are treated as a combined residual, $R_{v}=\left\{R_{v, r}, R_{v, c}, R_{v, \text { tot }}\right\}$, in evaluating a log-likelihood function as in Equation (1) (now the summation is over $v$ ). The same MCMC algorithm utilized in Section 3.1 was employed to optimize the model and sample the posterior PDFs for the model parameters. Because there are more parameters than constraints describing the point source, we adopted Gaussian priors on $\left\{\log S_{v, 0}^{\text {dust }}, \alpha_{\text {dust }}, \log S_{v, 0}^{\mathrm{ff}}, \alpha_{\mathrm{ff}}\right\}$, with means $\{-4.65,2.1,-4.25$, $0.0\}$ and widths $\{0.2,0.2,0.2,0.1\}$ (the normalizations are in $\log$ Jy units) informed by the examination of the $S_{v, c}$ spectrum described above. To incorporate the measurements of the resolved emission structure found in Section 3.1, we assumed Gaussian priors for $\left\{\mu_{r}, \sigma_{r}, i\right\}$ with means $\{235 \mathrm{AU}, 25 \mathrm{AU}$, $\left.37^{\circ}\right\}$ and widths $\left\{5 \mathrm{AU}, 5 \mathrm{AU}, 1^{\circ}\right\}$ (see Table 2 ). Uniform priors were assumed for the other parameters, $\left\{T_{r}, \Sigma_{0}, a_{\max }, q\right\}$.

The parameter inferences from these fits are summarized in Table 3 . The corresponding model spectra are compared with the data in Figure 5. In terms of the physical conditions in the ring, we infer a dust temperature of 20-30 K, comparable to the $35 \mathrm{~K}$ determined from the $\mathrm{CO}$ spectral line emission by Guilloteau et al. (1999). The difference is plausibly a manifestation of the modest temperature inversion that would be expected between the cooler midplane (where the dust emission is generated) and the warmer atmosphere traced by the CO (e.g., Dartois et al. 2003; Rosenfeld et al. 2013a). The dust surface density at the peak of the ring is only $0.01-0.03 \mathrm{~g} \mathrm{~cm}^{-2}$, implying a total dust mass of $\sim 30-90 M_{\oplus}$ (or $\left.1-3 \times 10^{-4} M_{\odot}\right)$. We find that relatively top-heavy grain size distributions, $q \approx 1.4-2.7$, provide substantially better fits than the typical assumptions based on models of collisional cascades (Dohnanyi 1969) or measurements in the diffuse interstellar medium (Mathis et al. 1977 ), where $q \approx 3.5$. With more mass concentrated near the maximum particle size, $a_{\max } \approx 1-2 \mathrm{~mm}$, the corresponding

\footnotetext{
19 Here we also add in quadrature a systematic calibration uncertainty term at
} each frequency; see Section 2. 


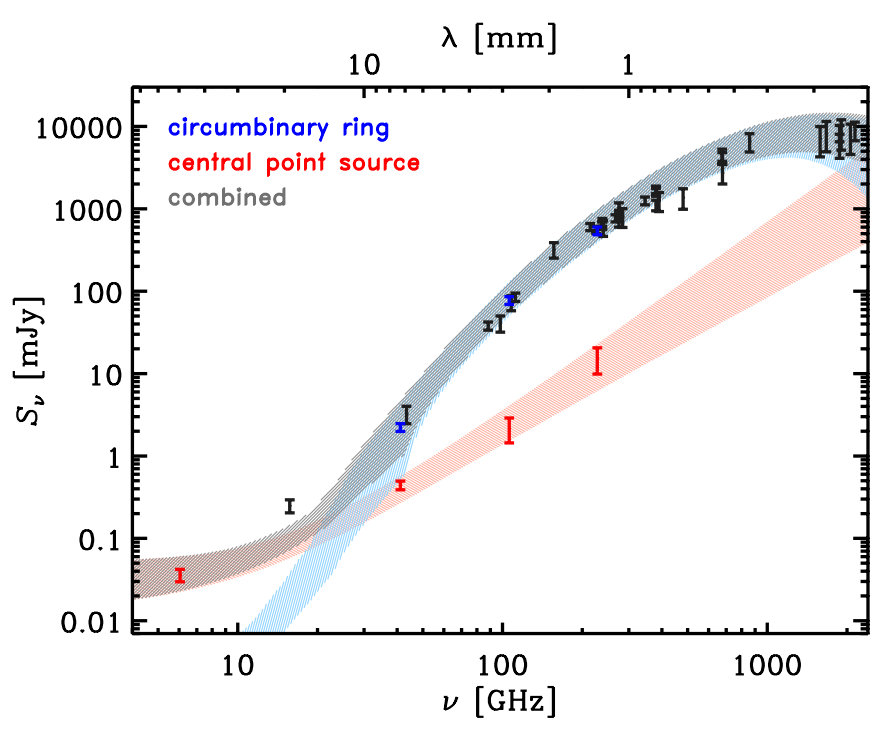

Figure 5. Far-infrared to radio spectrum of GG Tau A, decomposed into the circumbinary ring (blue) and central point source (red), along with their combination (gray). The model spectrum contributions from each component, based on random draws from the posterior PDF, are overlaid on the data; their widths represent the $95 \%(\sim 2 \sigma)$ confidence boundaries.

(A color version of this figure is available in the online journal.)

Table 3

Inferred Spectrum Model Parameters

\begin{tabular}{lc}
\hline \hline Parameter & Best-fit Value $( \pm 1 \sigma)$ \\
\hline$\Sigma_{0}\left(\mathrm{~g} \mathrm{~cm}^{-2}\right)$ & $0.02 \pm 0.01$ \\
$\mu_{r}(\mathrm{AU})$ & $(235 \pm 5)$ \\
$\sigma_{r}(\mathrm{AU})$ & $(25 \pm 5)$ \\
$T_{r}(\mathrm{~K})$ & $25 \pm 5$ \\
$a_{\max }(\mathrm{mm})$ & $1.5_{-0.9}^{+0.3}$ \\
$q$ & $2.4_{-1.0}^{+0.3}$ \\
$\log S_{v, 0}^{\text {dust }}(\mathrm{Jy})$ & $(-4.65 \pm 0.20)$ \\
$\alpha^{\text {dust }}$ & $(2.1 \pm 0.2)$ \\
$\log S_{v, 0}^{\mathrm{ff}}(\mathrm{Jy})$ & $(-4.50 \pm 0.20)$ \\
$\alpha^{\text {ff }}$ & $(0.0 \pm 0.1)$ \\
\hline
\end{tabular}

Notes. These parameters are valid for the Ricci et al. (2010b) dust mixture with 30\% porosity. See the text for a discussion of alternative assumptions. The values in parenthesis reflect the Gaussian priors we assumed for these fits. The formal reduced $\chi^{2}$ statistic for the best-fit model is $\sim 1.8$ (see the text).

dust opacity spectrum ends up having substantial curvature at wavelengths near $a_{\max }$, reproducing well the observed shape of the mm-wave ring spectrum. A reconstruction of the inferred $\kappa_{v}$ is shown in Figure 6: the $1.3 \mathrm{~mm}$ opacity lies in the range of 5-10 $\mathrm{cm}^{2} \mathrm{~g}^{-1}, \sim 2-4$ times larger than the standard Beckwith et al. (1990) opacity prescription for disks. Note that the ring is optically thin at all frequencies of interest here.

The best-fit model has a $\chi^{2} \approx 62$, and a reduced $\tilde{\chi}^{2} \approx 1.8$ (46 datapoints, 11 free parameters). ${ }^{20}$ Some of these residuals are likely due to under-estimates of flux density uncertainties, particularly for older single-dish photometers at challenging submillimeter wavelengths. It is worth explicitly pointing out that the favored models systematically underpredict the

\footnotetext{
${ }^{20}$ Note that we do not double-count the ring + central source photometry measured here in the "combined" model fit, despite listing those measurements in Table 1 (for the sake of completeness).
}

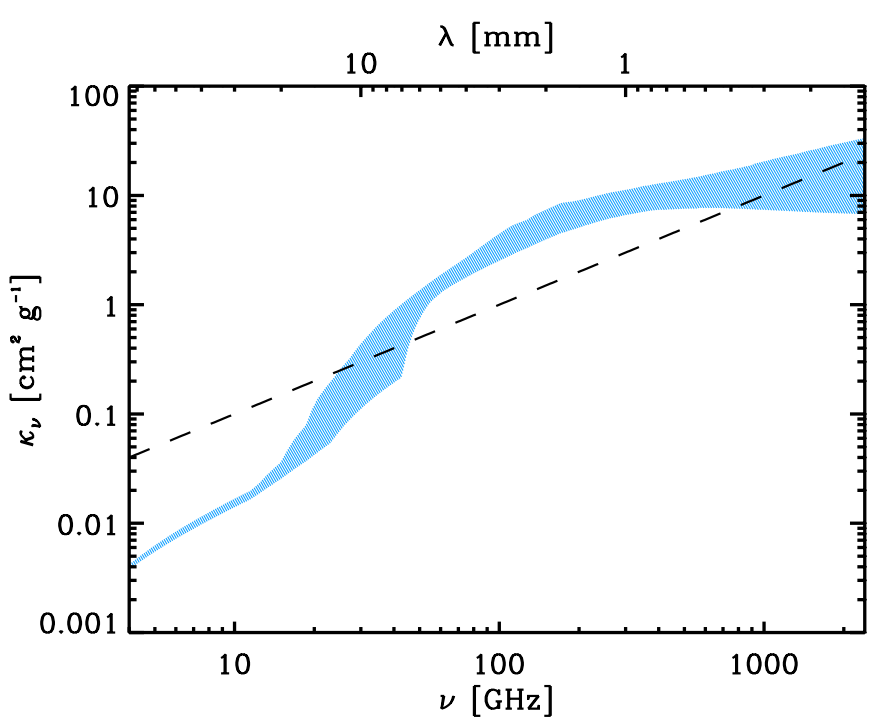

Figure 6. Constraints on the dust opacity spectrum $(\sim 2 \sigma$ confidence intervals) in the GG Tau A circumbinary ring, reconstructed from random draws of the joint posterior PDF derived from modeling the far-infrared to radio spectrum. Overlaid as a dashed curve is the standard opacity prescription used for protoplanetary disks, originally advocated by Beckwith et al. (1990).

(A color version of this figure is available in the online journal.)

$K u$-band (16 GHz) flux density reported by Scaife (2013) at the $\sim 3 \sigma$ level (this datapoint alone drives $\chi^{2}$ up by $\sim 10$ ). Perhaps this is due to the difficulty of disentangling the GG Tau $\mathrm{A}$ and $\mathrm{N}$ emission in those data, ${ }^{21}$ where the resolution was nearly three times the A-N separation and the contrast ratio is high (in her Figure 1, Scaife indicates that $\mathrm{N}$ is roughly an order of magnitude brighter than A at this frequency). Alternatively, it is possible that the measurement uncertainties are fine, and instead some of the assumptions made in the modeling are responsible for the inferred (modest) residuals. To explore that possibility further, we re-fit the data with several modifications to our critical assumptions.

In motivating the simplicity of the GG Tau A circumbinary ring structure, we suggested that its dust temperature is roughly constant. However, a radial temperature gradient could be present, particularly if the inner part of the ring intercepts a substantial amount of incident irradiation from the central stars (analogous to the dust sublimation boundaries of most disks, or the "wall" features just outside transition disk cavities; e.g., Dullemond et al. 2001; Calvet et al. 2002). As an extreme counter-example, we re-modeled the spectrum assuming that the dust temperatures vary inversely with radius in the ring $\left(T_{r} \propto 1 / r\right)$. No significant differences in the model parameters were found, as might be expected given the narrowness of the ring. This check validates the simplified assumption that reasonable temperature gradients have negligible impact on our results.

The model optimization described above currently requires relatively stringent priors on the central point source parameters (given the sparse data). But there is considerable leeway in those prior assumptions that could still offer reasonable fits to the data. To assess the impact of these priors on the main physical parameters of the circumbinary ring, we re-modeled the spectrum with a steeper combination of Gaussian priors on the spectral indices, with means $\left\{\alpha_{\text {dust }}, \alpha_{\mathrm{ff}}\right\}=\{2.5,1.0\}$, and adjustments to their corresponding normalizations (at $10 \mathrm{GHz}$ ),

\footnotetext{
${ }^{21}$ As well as any (relatively) small contribution from GG Tau B.
} 
$\left\{\log S_{v, 0}^{\text {dust }}, \log S_{v, 0}^{\mathrm{ff}}\right\}=\{-5.25,-4.25\}$ (in Jy units; the widths of the priors were the same as used above). We find that adopting these alternative priors has no notable effect on the key ring parameters; the point source is simply too faint to matter in this regard (nor does it improve the fit quality, even for the $K u$-band point noted above). That said, the uncertain origins of the central point source emission will remain an open question until resolved measurements at additional frequencies are available.

Perhaps the more relevant assumptions made in the modeling concern the nature of the grains themselves, particularly their porosities and material compositions. To investigate these issues further, we first re-modeled the GG Tau A spectrum assuming different (fixed) porosities. Compared to our nominal parameters $(30 \%$ porosity), models with "compact" grains $(0 \%$ porosity) can explain the data reasonably well if the circumbinary ring has a similar $T_{r}$, a slightly $(\sim 30 \%-50 \%)$ lower dust mass, and a steeper size distribution $(q \approx 2.5-3.7)$ with $a_{\max }$ just under $1 \mathrm{~mm}$. For higher porosities $(60 \%)$, we instead infer a $(5-8 \times)$ higher dust mass and shallow size distribution $(q \approx 1.0-2.8)$ up to a maximum size of a few $\mathrm{mm}$ to $\sim 1 \mathrm{~cm}$. Since $a_{\max }$ is comparable to the wavelengths of interest, resonances preferentially enhance the millimeter-wave opacities for grains with higher filling factors (lower porosities; e.g., Miyake \& Nakagawa 1993; Kataoka et al. 2013, but see Cuzzi et al. 2014): a higher $\kappa_{v}$ drives us to find lower $\Sigma_{0}$ (and therefore dust mass) for a fixed spectrum (and vice versa). These same resonances also naturally introduce an intrinsic curvature into the millimeter/centimeter-wave opacity spectrum that is reflected in the inferred size distribution index $(q)$ : since compact grains then do not require the added $\kappa_{v}$ curvature produced by a topheavy size distribution, it makes sense that we infer a higher $q$ when the porosity is low (and vice versa). For a fixed composition, grains with $\sim 20 \%-40 \%$ porosity do produce better fits to the data.

Next, we performed a similar experiment that varied the material composition of the grains, by re-fitting the data with extreme silicate- or carbon-rich mixtures. ${ }^{22}$ Compared to our adopted mixture (with $7 \%$ silicates and $21 \%$ carbonaceous material by volume), models with $\mathrm{C}$-rich grains (28\% carbon, no silicates) in the GG Tau A ring have a comparable dust mass and temperature, and a modestly steeper grain size distribution $(q \approx 2.2-3.5)$ up to $a_{\max } \approx 1 \mathrm{~mm}$. In the opposite case, models with Si-rich grains tend toward $(\sim 10 \times)$ higher dust masses and shallow size distributions $(q \approx 1.1-2.6)$ with $a_{\max } \approx 2-8 \mathrm{~mm}$. Silicate-rich grains have $\mathrm{mm} / \mathrm{cm}$-wave opacity spectra that exhibit less curvature and are preferentially reduced compared to C-rich grains, properties that naturally account for our inferences of top-heavy size distributions and higher dust masses when $\mathrm{C}$ is depleted, respectively. That said, models relying on these extreme compositions produce poor fits to the data; the experiment is only intended to convey the sense of the variation.

While there are many alternative assumptions along these lines that could be made in the modeling, the investigations described above demonstrate that most of the key parameters related to the dust in the GG Tau A circumbinary ring are relatively robust. Within a reasonably large range of temperature gradients, point source emission models, grain porosities and com-

\footnotetext{
22 Since water ice plays only a minor role relative to silicates and carbonaceous material in setting $\kappa_{v}$ in the $\mathrm{mm} / \mathrm{cm}$ wavelength range, its volume fraction is left fixed (at 42\%; e.g., Pollack et al. 1994) in this experiment.
}

positional variations, we find dust temperatures of $\sim 20-30 \mathrm{~K}$, dust masses of $\sim 1-10 \times 10^{-4} M_{\odot}$, and maximum grain sizes of 1-10 $\mathrm{mm}$ (in all cases with preference near the lower end of the quoted ranges). However, it should be clear that these data do not provide a strong quantitative constraint on the power-law index of the grain size distribution, $q$. Finally, as a point of reference, we find that the dust opacity at $1.3 \mathrm{~mm}$ is restricted to $\sim 0.2-20 \mathrm{~cm}^{2} \mathrm{~g}^{-1}$ in all the models explored here.

\section{DISCUSSION AND CONCLUSIONS}

We have obtained and analyzed high angular resolution observations of continuum emission associated with GG Tau, a young quadruple star system, at wavelengths of 1.3, 2.8, 7.3 , and $50 \mathrm{~mm}$. These data confirm that GG Tau/N is a background synchrotron source, and identify faint $7.3 \mathrm{~mm}$ emission associated with the low-mass star GG Tau Ba, although the origin of the latter is unclear. As had been noted previously (e.g., Guilloteau et al. 1999), we find a bright emission ring and central peak associated with GG Tau A (although the ring is undetected at a wavelength of $5 \mathrm{~cm}$ ). The visibility data were modeled with a simple surface brightness prescription. We found that the emission at all frequencies could be described well using a (slightly offset) central point source and a (projected) circular Gaussian ring with a mean radius $\left(\mu_{r}\right)$ of $235 \pm 5 \mathrm{AU}$ and width $\left(\sigma_{r}\right)$ of $25 \pm 5 \mathrm{AU}$ (a FWHM of $60 \pm 10 \mathrm{AU}$ ), reasonably consistent with previous constraints based on slightly different assumptions (Dutrey et al. 1994; Guilloteau et al. 1999; Piétu et al. 2011). These morphological constraints indicate that there is negligible radial variation (on angular scales $\gtrsim 0$ '. 1 ) of the $\mathrm{mm} / \mathrm{cm}$-wavelength spectrum $(\alpha \lesssim 0.3$, from $1.3-7.3 \mathrm{~mm}$ ) across the circumbinary ring.

The spectrum of the point source flattens considerably at the longest radio wavelengths, suggesting emission contributions from both dust and free-free radiation. The integrated spectrum of the ring structure exhibits substantial curvature, becoming steeper at longer wavelengths, which we suggest is a manifestation of the intrinsic shape of the dust opacity spectrum. We developed some simple physical models of the observed GG Tau A spectrum, and found reasonably good fits for dust temperatures $\left(T_{r}\right)$ of $20-30 \mathrm{~K}$, dust masses of $30-60 M_{\oplus}\left(1-3 \times 10^{-4} M_{\odot}\right)$, and relatively top-heavy grain size distributions $\left(d n / d a \propto a^{-q}\right.$, with $q \approx 1.4-2.7)$ up to maximum particle sizes $\left(a_{\max }\right)$ of 1-2 mm, for the grain composition of Ricci et al. (2010b; effectively the Pollack et al. 1994 mixture with $30 \%$ porosity). Alternative assumptions about the mineralogical makeup and porosities of the grains permit a much wider range of the size index $q$, but still suggest similar temperatures and a relatively narrow range of masses $\left(\sim 1-10 \times 10^{-4} M_{\odot}\right)$ and maximum particle sizes $(\sim 1-10 \mathrm{~mm})$. In any case, the opacity spectrum inferred in the ring is not described well with a single power-law in the $\mathrm{mm} / \mathrm{cm}$ wavelength range; the standard assumption that $\kappa_{v} \propto v^{\beta}$ is not appropriate here. Although we have estimated significantly lower masses (up to a factor of $\sim 5$ compared to, e.g., Guilloteau et al. 1999; Andrews \& Williams 2005) and maximum grain sizes $(\sim 4-40$ times smaller than inferred by Scaife 2013) in the ring than in previous studies, these properties still seem remarkably high given the large separation from the central stars, narrow radial width of the ring, and age of the system ( 1-3 Myr; or rather time available for particle growth).

A promising way to concentrate and grow dust particles up to $\sim$ millimeter sizes at such large distances, as well as to halt their normally fast inward migration due to radial drift, relies on creating a "trap" in a local enhancement of the gas pressure 


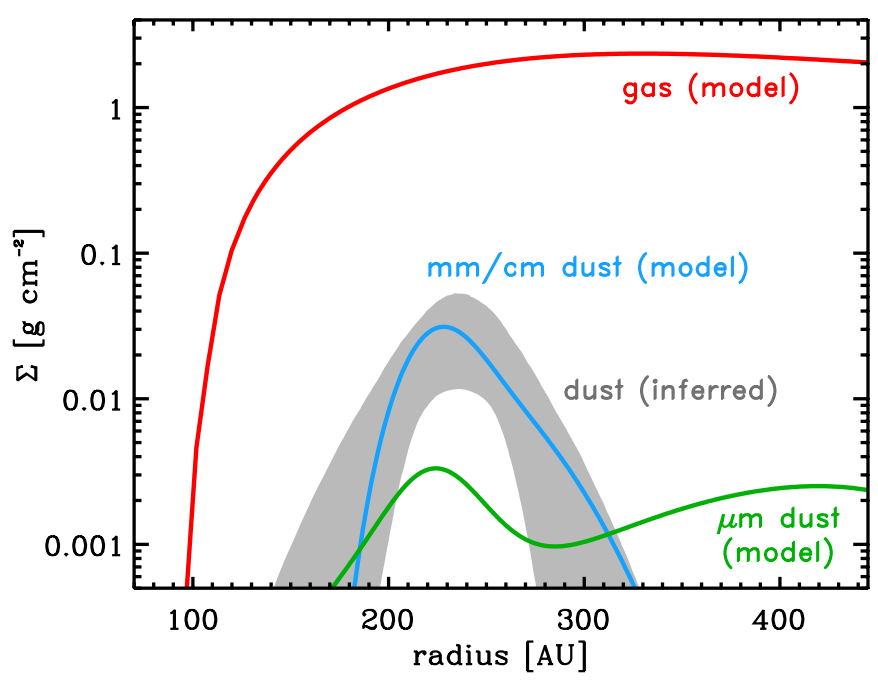

Figure 7. Schematic snapshot for an illustrative model of dust evolution in a truncated gas disk with a gradually tapered inner edge, tuned to the relevant parameters of the GG Tau A circumbinary disk. We assumed a static power-law gas surface density profile ( $\Sigma_{\text {gas }} \propto 1 / r$; red curve), with an "edge" near the circumbinary disk truncation radius expected from estimates of the GG Tau A orbit, and a gradual Gaussian taper (with mean $100 \mathrm{AU}$ and width $120 \mathrm{AU}$ ). The total gas mass was $0.13 M_{\odot}(\sim 10 \%$ of the binary mass), consistent with the estimate of Guilloteau et al. (1999). A population of small $(0.1-1 \mu \mathrm{m}$, with $\left.d n / d a \propto a^{-3.5}\right)$ grains with radially constant dust-to-gas mass ratio (0.01) was evolved in size and space for $1 \mathrm{Myr}$, following the Birnstiel et al. (2010) prescription for a constant turbulent viscosity parameter $(\alpha=0.002)$ and fragmentation velocity $\left(10 \mathrm{~m} \mathrm{~s}^{-1}\right)$. The surface density distributions of $\mu \mathrm{m}$ sized (0.5-5 $\mu \mathrm{m}$; green) and $\mathrm{mm} / \mathrm{cm}$-sized (500 $\mu \mathrm{m}<a<5 \mathrm{~cm}$; blue) particles predicted by this model are in reasonably good agreement with both infrared scattered light observations (e.g., Duchêne et al. 2004) and our estimates of $\Sigma_{\text {dust }}$ inferred from the modeling in Section 3 (the gray shaded region corresponds to the $2 \sigma$ uncertainties).

(A color version of this figure is available in the online journal.)

profile (e.g., Whipple 1972). For GG Tau A, this local pressure maximum would likely be induced by dynamical interactions between the binary and disk edge (e.g., Artymowicz \& Lubow 1994). However, models of the GG Tau A stellar orbit highlight a potential issue with this interpretation, since the ring edge predicted by dynamical simulations lies well inside (at a radius of roughly $\sim 100 \mathrm{AU}$ ) the edge location inferred from dust observations (e.g., McCabe et al. 2002; Beust \& Dutrey 2005; Köhler 2011). Pinilla et al. (2012a) have suggested that a dust trap could reside substantially beyond the nominal disk "edge" if the gas pressures decrease (relatively) gradually toward the inner part of the ring. To schematically illustrate this point in the case of GG Tau, we used the treatment of Birnstiel et al. (2010) to simulate the evolution of dust particles - both spatially and in size, subject to growth, fragmentation, viscous diffusion, and radial drift - in a gas disk that has a (static) power-law surface density profile with a Gaussian tapered edge at a radius of 100 AU. Tuning the shape of this taper (i.e., the width of the Gaussian) can push the gas pressure maximum back to a radius comparable to the observed dust ring location. For a reasonable turbulent viscosity parameter $(\alpha \approx 0.002)$ and density normalization (a total gas mass of $\sim 0.1 M_{\odot}$ ), we can also reproduce the inferred maximum particle size and width of the dust ring on appropriate timescales $(\sim 1 \mathrm{Myr})$. This simulation is also in qualitative agreement with the infrared scattered light geometry of the GG Tau A ring (e.g., Duchêne et al. 2004), in that it predicts a similar distribution of micron-sized grains produced via fragmentation in the pressure maximum. Figure 7 summarizes this demonstrative (although by no means unique) example.

Of course, it is not clear if such "soft" edges are physically realistic for gas-rich circumbinary disks like the one around GG Tau A; detailed hydrodynamic simulations (e.g., Bate 2000; Günther \& Kley 2002; Kley et al. 2008) tuned to this specific case would be required to assess the feasibility and internal consistency of the basic scenario proposed above (although see Beust \& Dutrey 2005). Regardless, the key point is that we should be actively considering the coupled evolution of gas and solids in this and similar disks when attempting to reconcile models of their tidal truncation with the (still uncertain) stellar orbital configurations. In principle, one could test the proposed explanation with sensitive observations of an optically thin line tracer that tracks the gas densities near and interior to the dust disk edge at $\sim 0$ '. 1 resolution. ${ }^{23}$ In that context, it is compelling to note that Guilloteau et al. (1999) found that the ${ }^{13} \mathrm{CO}$ emission extends slightly inside the continuum ring in their study of the GG Tau A disk; similar data at higher resolution would be valuable. These dust trap models also predict that larger particles should be concentrated at the maximum pressure, although the implied spectral gradient in this particular example would not be resolved with our data. In that sense, pushing the angular resolution of multifrequency continuum observations in this and other cases should be considered a high priority.

Although the GG Tau A ring is a particularly spectacular (and useful) example, the dust in circumbinary disks more generally could serve as important test cases for studying particle traps induced by dynamical interactions with companions. The socalled "transition" disks, where the companion is speculated to be a giant planet, exhibit similar features-narrow dust continuum rings (e.g., Andrews et al. 2011), often having more extended gas disks with relatively lower dust masses (e.g., Isella et al. 2012; Rosenfeld et al. 2013b) and occasionally showing direct (Rosenfeld et al. 2012, 2014; Casassus et al. 2013; van der Marel et al. 2013; Bruderer et al. 2014) or indirect (e.g., Dong et al. 2012; Follette et al. 2013) evidence for gas well inside those dust rings. Given that similarity, we suggest that there is great value in analyzing resolved multifrequency radio observations of dust rings (and gas structures) in both circumbinary and transition disks, analogous to the approach presented here. Ultimately, linking these disk targets-which are undergoing dynamical interactions with a broad range of companion masses-could offer important insights on how solids grow inside gas pressure maxima with a wide diversity of strengths and shapes.

We thank Mark Reid for valuable discussions about data modeling and an anonymous referee for helpful suggestions. S.M.A. and T.B. acknowledge support from NASA Origins of Solar Systems grant NNX12AJ04G. A.I., L.M.P., and J.M.C. acknowledge support from NSF award AST-1109334. Ongoing CARMA development and operations are supported by the National Science Foundation under a cooperative agreement, and by the CARMA partner universities. The VLA is run by the National Radio Astronomy Observatory, a facility of the National Science Foundation operated under cooperative agreement by Associated Universities, Inc.

\footnotetext{
23 It is worth noting that this would also require a proper accounting of the gas temperatures, which might not be trivial in such a relatively dust-poor environment (see Bruderer 2013).
} 


\section{REFERENCES}

Adachi, I., Hayashi, C., \& Nakazawa, K. 1976, PThPh, 56, 1756

Adams, F. C., Lada, C. J., \& Shu, F. H. 1987, ApJ, 312, 788

Andrews, S. M., Rosenfeld, K. A., Kraus, A. L., \& Wilner, D. J. 2013, ApJ, 771,129

Andrews, S. M., \& Williams, J. P. 2005, ApJ, 631, 1134

Andrews, S. M., Wilner, D. J., Espaillat, C., et al. 2011, ApJ, 732, 42

Andrews, S. M., Wilner, D. J., Hughes, A. M., et al. 2012, ApJ, 744, 162

Artymowicz, P., \& Lubow, S. H. 1994, ApJ, 421, 651

Artymowicz, P., \& Lubow, S. H. 1996, ApJL, 467, L77

Banzatti, A., Testi, L., Isella, A., et al. 2011, A\&A, 525, A12

Bate, M. R. 2000, MNRAS, 314, 33

Beckwith, S. V. W., \& Sargent, A. I. 1991, ApJ, 381, 250

Beckwith, S. V. W., Sargent, A. I., Chini, R. S., \& Guesten, R. 1990, AJ, 99, 924

Beust, H., \& Dutrey, A. 2005, A\&A, 439, 585

Bieging, J. H., Cohen, M., \& Schwartz, P. R. 1984, ApJ, 282, 699

Birnstiel, T., \& Andrews, S. M. 2014, ApJ, 780, 153

Birnstiel, T., Dullemond, C. P., \& Brauer, F. 2010, A\&A, 513, A79

Birnstiel, T., Klahr, H., \& Ercolano, B. 2012, A\&A, 539, A148

Brauer, F., Dullemond, C. P., Johansen, A., et al. 2007, A\&A, 469, 1169

Bruderer, S. 2013, A\&A, 559, A46

Bruderer, S., van der Marel, N., van Dishoeck, E. F., \& van Kempen, T. A. 2014, A\&A, 562, A26

Calvet, N., D'Alessio, P., Hartmann, L., et al. 2002, ApJ, 568, 1008

Casassus, S., van der Plas, G., M, S. P., et al. 2013, Natur, 493, 191

Cranmer, S. R., Wilner, D. J., \& MacGregor, M. A. 2013, ApJ, 772, 149

Cuzzi, J. N., Estrada, P. R., \& Davis, S. S. 2014, ApJS, 210, 21

D’Alessio, P., Calvet, N., \& Hartmann, L. 2001, ApJ, 553, 321

Dartois, E., Dutrey, A., \& Guilloteau, S. 2003, A\&A, 399, 773

Dohnanyi, J. S. 1969, JGR, 74, 2531

Dong, R., Rafikov, R., Zhu, Z., et al. 2012, ApJ, 750, 161

Draine, B. T. 2006, ApJ, 636, 1114

Duchêne, G., McCabe, C., Ghez, A. M., \& Macintosh, B. A. 2004, ApJ, 606, 969

Dullemond, C. P., Dominik, C., \& Natta, A. 2001, ApJ, 560, 957

Dutrey, A., Guilloteau, S., \& Simon, M. 1994, A\&A, 286, 149

Dzyurkevich, N., Flock, M., Turner, N. J., Klahr, H., \& Henning, T. 2010, A\&A, 515, A70

Follette, K. B., Tamura, M., Hashimoto, J., et al. 2013, ApJ, 767, 10

Foreman-Mackey, D., Hogg, D. W., Lang, D., \& Goodman, J. 2013, PASP, 125,306

Ghez, A. M., Neugebauer, G., \& Matthews, K. 1993, AJ, 106, 2005

Goodman, J., \& Weare, J. 2010, Commun. Appl. Math. Comput. Sci., 5, 65

Guilloteau, S., Dutrey, A., Piétu, V., \& Boehler, Y. 2011, A\&A, 529, A105

Guilloteau, S., Dutrey, A., \& Simon, M. 1999, A\&A, 348, 570

Günther, R., \& Kley, W. 2002, A\&A, 387, 550

Harris, R. J., Andrews, S. M., Wilner, D. J., \& Kraus, A. L. 2012, ApJ, 751,115

Hayasaki, K., \& Okazaki, A. T. 2009, ApJL, 691, L5

Henning, T., \& Stognienko, R. 1996, A\&A, 311, 291

Howard, C. D., Sandell, G., Vacca, W. D., et al. 2013, ApJ, 776, 21

Isella, A., Carpenter, J. M., \& Sargent, A. I. 2010, ApJ, 714, 1746

Isella, A., Pérez, L. M., \& Carpenter, J. M. 2012, ApJ, 747, 136

Itoh, Y., Tamura, M., Hayashi, S. S., et al. 2002, PASJ, 54, 963

Johansen, A., Blum, J., Tanaka, H., et al. 2014, in Protostars \& Planets VI, ed.

H. Beuther, R. Klessen, C. Dullemond, \& Th. Henning (Tucson, AZ: Univ. Arizona Press), in press (arXiv:1402.1344)

Kataoka, A., Okuzumi, S., Tanaka, H., \& Nomura, H. 2013, arXiv:1312.1459

Klahr, H. H., \& Henning, T. 1997, Icar, 128, 213

Kley, W., \& Dirksen, G. 2006, A\&A, 447, 369
Kley, W., Papaloizou, J. C. B., \& Ogilvie, G. I. 2008, A\&A, 487, 671

Köhler, R. 2011, A\&A, 530, A126

Krist, J. E., Stapelfeldt, K. R., Golimowski, D. A., et al. 2005, AJ, 130,2778

Krist, J. E., Stapelfeldt, K. R., \& Watson, A. M. 2002, ApJ, 570, 785

Leinert, C., Haas, M., Mundt, R., Richichi, A., \& Zinnecker, H. 1991, A\&A, 250,407

Looney, L. W., Mundy, L. G., \& Welch, W. J. 2000, ApJ, 529, 477

Luhman, K. L., Allen, P. R., Espaillat, C., Hartmann, L., \& Calvet, N. 2010, ApJS, 186, 111

Mathis, J. S., Rumpl, W., \& Nordsieck, K. H. 1977, ApJ, 217, 425

McCabe, C., Duchêne, G., \& Ghez, A. M. 2002, ApJ, 575, 974

Menu, J., van Boekel, R., Henning, T., et al. 2014, A\&A, 564, 93

Miyake, K., \& Nakagawa, Y. 1993, Icar, 106, 20

Moriarty-Schieven, G. H., \& Butner, H. M. 1997, ApJ, 474, 768

Moriarty-Schieven, G. H., Wannier, P. G., Keene, J., \& Tamura, M. 1994, ApJ, 436, 800

Ohashi, N., Kawabe, R., Ishiguro, M., \& Hayashi, M. 1991, AJ, 102, 2054

Panić, O., Hogerheijde, M. R., Wilner, D., \& Qi, C. 2009, A\&A, 501, 269

Pérez, L. M., Carpenter, J. M., Chandler, C. J., et al. 2012, ApJL, 760, L17

Perley, R. A., \& Butler, B. J. 2013, ApJS, 204, 19

Piétu, V., Gueth, F., Hily-Blant, P., Schuster, K.-F., \& Pety, J. 2011, A\&A, 528, A81

Pinilla, P., Benisty, M., \& Birnstiel, T. 2012a, A\&A, 545, A81

Pinilla, P., Birnstiel, T., Ricci, L., et al. 2012b, A\&A, 538, A114

Pollack, J. B., Hollenbach, D., Beckwith, S., et al. 1994, ApJ, 421, 615

Reynolds, S. P. 1986, ApJ, 304, 713

Ricci, L., Testi, L., Natta, A., \& Brooks, K. J. 2010a, A\&A, 521, A66

Ricci, L., Testi, L., Natta, A., et al. 2010b, A\&A, 512, A15

Roddier, C., Roddier, F., Northcott, M. J., Graves, J. E., \& Jim, K. 1996, ApJ, 463,326

Rodmann, J., Henning, T., Chandler, C. J., Mundy, L. G., \& Wilner, D. J. 2006, A\&A, 446, 211

Rosenfeld, K. A., Andrews, S. M., Hughes, A. M., Wilner, D. J., \& Qi, C. 2013a, ApJ, 774, 16

Rosenfeld, K. A., Andrews, S. M., Wilner, D. J., Kastner, J. H., \& McClure, M. K. 2013b, ApJ, 775, 136

Rosenfeld, K. A., Chiang, E., \& Andrews, S. M. 2014, ApJ, 782, 62

Rosenfeld, K. A., Qi, C., Andrews, S. M., et al. 2012, ApJ, 757, 129

Scaife, A. M. M. 2013, MNRAS, 435, 1139

Silber, J., Gledhill, T., Duchêne, G., \& Ménard, F. 2000, ApJL, 536, L89

Sivia, D. S., \& Skilling, J. 2006, Data Analysis: A Bayesian Tutorial (2nd ed.; New York: Oxford Univ. Press), 168

Takeuchi, T., \& Lin, D. N. C. 2002, ApJ, 581, 1344

Takeuchi, T., \& Lin, D. N. C. 2005, ApJ, 623, 482

Testi, L., Birnstiel, T., Ricci, L., et al. 2014, in Protostars \& Planets VI, ed. H. Beuther, R. Klessen, C. Dullemond, \& Th. Henning (Tucson, AZ: Univ. Arizona Press), in press (arXiv:1402.1354)

Torres, R. M., Loinard, L., Mioduszewski, A. J., \& Rodríguez, L. F. 2009, ApJ, 698,242

Trotta, F., Testi, L., Natta, A., Isella, A., \& Ricci, L. 2013, A\&A, 558, A64

van der Marel, N., van Dishoeck, E. F., Bruderer, S., et al. 2013, Sci, 340,1199

Warren, S. G. 1984, ApOpt, 23, 1206

Weidenschilling, S. J. 1977, MNRAS, 180, 57

Weingartner, J. C., \& Draine, B. T. 2001, ApJ, 548, 296

Whipple, F. L. 1972, in From Plasma to Planet, ed. A. Elvius (New York: Wiley), 211

White, R. J., Ghez, A. M., Reid, I. N., \& Schultz, G. 1999, ApJ, 520, 811

White, S. M., Lim, J., \& Kundu, M. R. 1994, ApJ, 422, 293

Zubko, V. G., Mennella, V., Colangeli, L., \& Bussoletti, E. 1996, MNRAS, 282,1321 